\title{
Ship tracking results from state-of-the-art space-based AIS receiver systems for maritime surveillance
}

\author{
Andreas Nordmo Skauen ${ }^{1}$ (D)
}

Received: 8 January 2019 / Revised: 21 March 2019 / Accepted: 31 March 2019 / Published online: 11 April 2019

(c) The Author(s) 2019

\begin{abstract}
Norway currently operates four satellites with Automatic Identification System (AIS) receivers. The first-generation satellites, AISSat-1 and AISSat-2, are equipped with a two-channel, single-antenna AIS receiver, while NorSat-1 and NorSat-2 are equipped with an improved AIS receiver capable of decoding on all four AIS channels and using the two antennas installed on the NorSat satellites. This paper aims to investigate the ship tracking performance enhancement realised by the technology improvements of antenna diversification, frequency diversification, and advanced algorithms. The ship tracking capability of the NorSat satellites is presented and shown to yield a significant improvement, up to a $20 \%$ point increase, over the first AISSat generation ship tracking capability. A further $20 \%$ point increase is achieved in select areas using frequency diversity introduced in the AIS system since the development of the AISSat satellites. In addition, NorSat-1 detected 34\% more vessels than AISSat-2 over the same timeframe. The contribution to the performance improvement from the incremental improvements in decoding algorithms, antenna diversity, and frequency diversity is indicated in the results. The results indicate that, in the short term, upgrading to the latest algorithms, low noise electronics, and taking advantage of antenna diversity is the greatest performance enhancer. In the medium and long term, the frequency diversity likely yields the greatest performance enhancement.
\end{abstract}

Keywords Automatic Identification System (AIS) - Space-based AIS · Maritime surveillance $\cdot$ Ship tracking $\cdot$ NorSat · AISSat

\section{Introduction}

Norway currently operates four satellites with Automatic Identification System (AIS) receivers. Since the launch of the first satellite in 2010, AISSat-1 [1,2], satellite AIS systems have gone from being an experimental service to being a fully operational capability with an unprecedented ability to monitor ship traffic on a global scale [3]. The significant value added by space-based AIS systems convinced Norwegian authorities to provide long-term funding of a space-based AIS program to continually replenish the space infrastructure and maintain the operational capability [4].

Work on more advanced algorithms has been published since the AISSat-1 launch in 2010 [5-7], as well as the use of multiple antennas to increase the probability of detecting messages that have experienced significant polarisation

Andreas Nordmo Skauen

Andreas-Nordmo.Skauen@ffi.no

1 Norwegian Defence Research Establishment (FFI), P.O.

Box 25, 2027 Kjeller, Norway rotation [8-12]. The rotation may reduce the signal strength required if using a receiving antenna of the same polarisation as the transmission antenna. Two, or three, cross-polarised monopole antennas will be able to account for more polarisation rotation of the messages, while, at the same time, individually suppressing overlapping messages of different polarisation rotation. Finally, the International Telecommunication Union (ITU) introduced two additional frequencies with a dedicated long-range message to the AIS standard, after the development of the AISSat-1 AIS receiver. The dedicated message was designed to improve detection probability for long-range applications such as space-based AIS systems. All AIS equipment must comply with the updated standard in time. For the purpose of this paper, the enhancement to the standard of two additional frequencies with a dedicated long-range message is referred to as frequency diversity.

The two most recently launched satellites in the Norwegian program, NorSat-1 and NorSat-2, implement all of the above technology improvements like more advanced decoding algorithms, antenna diversity, and frequency diversity. This paper aims to quantify the ship tracking capability 
of the NorSat-1 and NorSat-2 satellites and compare their ship tracking capability with that of the previous generation Norwegian satellites, AISSat-1 and AISSat-2, with an aim to investigate which of the technology improvements provide the greatest performance enhancement, as illustrated in Fig. 1.

There are many other space-based AIS systems currently in operation in addition to the Norwegian assets, ranging from commercial companies such as ORBCOMM and exactEarth [13, 14], to institutional initiatives such as the German Aerospace Centre (DLR) AIsat satellite [15] and the Japan Aerospace Exploration Agency (JAXA) SDS-4 satellite [16] to name a few of the many in each category.

The remainder of Sect. 1 briefly introduces the AIS system before providing a description of the four Norwegian satellites, their AIS payloads, and the notable differences between these. Section 2 describes how the ship tracking capability of the different systems is estimated. The resulting ship tracking capability of a single satellite, NorSat-1, and the combined capability of the NorSat- 1 and NorSat- 2 satellites are presented in Sect. 3. The capability when operating both on the nominal AIS1 and AIS2 channels and on the AIS3 and AIS4 channels, with the dedicated long-range AIS message, is presented. Section 4 presents the improved ship tracking capability realised by the aforementioned technology improvements by comparing the first-generation AISSat-2 capability with NorSat-1 over a common timeframe and vessel data set. All results are based on data gathered in January 2018. Finally, the presented results are discussed in Sect. 5 and a summary is presented in Sect. 6.

\subsection{AIS system}

The AIS system is fully described in the International Telecommunication Union recommendation ITU-R M.1371 [17]. AIS was introduced as a ship-to-ship and ship-to-shore reporting system intended to increase the safety of life at sea and to improve control and monitoring of maritime traffic. In version 4 of the standard, the reception of AIS messages by satellites was also included. AIS equipped ships broadcast their identity, position, speed, heading, cargo, destination, etc., to ships and shore stations within range of the VHF transmission. The nominal AIS channels, also known as AIS1 and AIS2, are $161.975 \mathrm{MHz}$ and $162.025 \mathrm{MHz}$. The channels intended for long-range use are $156.775 \mathrm{MHz}$ and $156.825 \mathrm{MHz}$, and are also known as AIS3 and AIS4. The long-range frequencies are referred to as the space AIS channels in this paper. Only vessels with updated equipment, following the latest version of the AIS standard, transmit on the space AIS channels.

The message content and reporting intervals depend on the type of message, and dynamic conditions of the ship such as speed and rate of turn. The ship tracking method and re-detection probability calculations presented in this
Fig. 1 Illustration of the aim of the paper: to quantify the improvement in ship tracking capability from the advanced algorithms and antenna and frequency diversification offered by the latest generation spacebased AIS systems
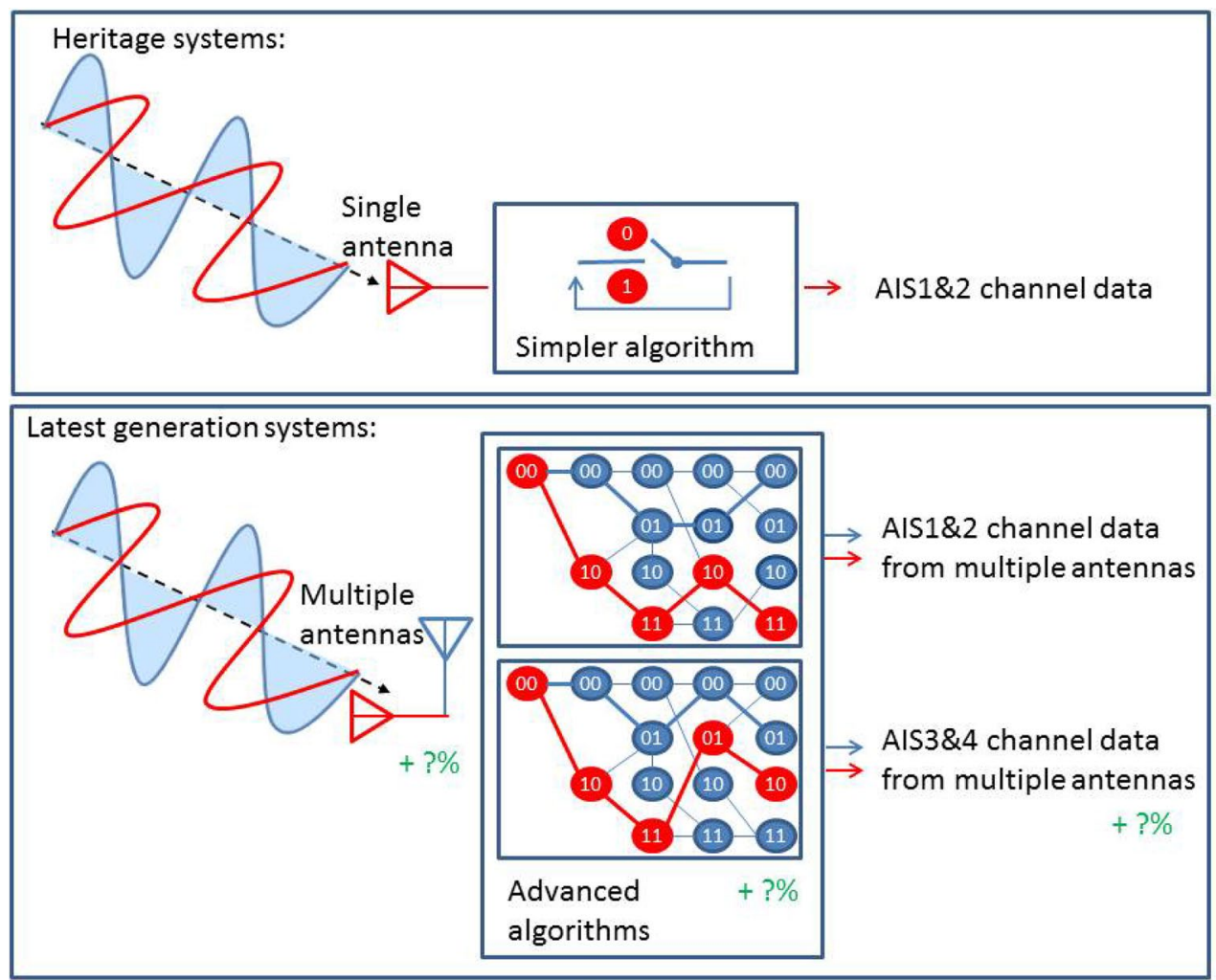
paper use message types $1-3$, transmitted on the nominal AIS channels, and type 27, transmitted on the space AIS channels. The message types 1-3 have dynamic content such as speed and position information, and are transmitted every $2-10 \mathrm{~s}$ depending on the specific dynamic conditions, e.g., speed and rate of turn. If stationary or moving slower than $3 \mathrm{knots}$, the reporting interval is $3 \mathrm{~min}$. The long-range type 27 message reporting interval is every 3 min regardless of dynamic conditions. The long-range message is reduced compared with the type 1-3 message types, but still contains position and speed information. All messages contain a unique identifier, the Maritime Mobile Service Identity (MMSI) number [18]. The MMSI number may change if the ship changes owner or any other registration details for instance, but is assumed to not change over the relatively short timescales investigated in this paper. Most AIS messages, including type 1-3 and 27, do not contain time information. However, knowing the time of transmission is critical for virtually all applications utilizing AIS data and space-based AIS receivers should affix a timestamp upon reception to approximate the time of transmission. Depending on the space-based AIS system ground station infrastructure, the AIS messages downloaded can be several hours old and the reception time becomes important for integration with coastal AIS data and/or other data. For the ship tracking method presented in this paper, it is further critical that the clock providing the timestamp to the space-based AIS system is correct to verify that the positions transmitted in the AIS messages are within the field of view of the satellite. Finally, all AIS messages also contain a repeat flag indicator that is set if the message has been repeated by an AIS transponder other than the message originator. This repeat flag indicator is important for the purposes of ship tracking in this paper, again because of the timing difference between original and repeated messages.

In high-vessel-density areas, such as the Mediterranean, Gulf of Mexico, North Sea, and East and South China Sea, for example, co-channel interference will degrade the performance of a space-based AIS system drastically [19]. In addition, there is evidence of strong land-based interference that also degrades the space-based AIS system performance when these interference sources are within the field of view [20]. Co-channel and land-based interference effectively raises the average noise level, leaving some signals with negative or too low signal-to-noise ratio for reliable reception for a specific AIS receiver. The long reporting interval of the long-range message on the space AIS channels was introduced specifically to increase the space-based AIS system capacity, since it reduces the cochannel interference [21]. However, the land-based interference will still cause significant problems when within the field of view of the space-based AIS system.

\subsection{Satellite and payload descriptions}

All four satellites, AISSat- 1 and -2 and NorSat- 1 and -2 , were built, tested, and prepared for flight by the Canadian Space Flight Laboratory at the University of Toronto Institute for Aerospace Studies (UTIAS/SFL) and launched as secondary "piggyback" satellites. The AIS receiver payloads were all developed and manufactured by Kongsberg Seatex AS, Trondheim, Norway. All the AIS payloads are software-defined radios that support optimisation of the receiver settings inorbit as well as uploading completely new payload algorithms, enabling higher performance during the lifetime if utilised. All the satellites were funded by the Norwegian Coastal Administration and Norwegian Space Agency in 2019 [4].

The AISSat-1 and AISSat- 2 satellites are for all practical purposes identical and carry an ASR100, single-antenna, two-channel software-defined radio AIS receiver. The satellites are $20 \times 20 \times 20 \mathrm{~cm}^{3}$, weighing approximately $6 \mathrm{~kg}$ with reaction wheels for full three-axis control. Both satellites use a single low-gain monopole antenna for AIS reception [1]. AISSat-1 was launched in 2010 and AISSat-2 in 2014, both into $\sim 98^{\circ}$ inclination, $\sim 620-\mathrm{km}$ altitude orbits. Both AISSat-1 and -2 AIS payload electronics are using upgraded algorithms based on development by the Norwegian Defence Research Establishment (FFI) and Kongsberg Seatex in a related European Space Agency project [22]. An on-average improved global performance of $25 \%$ with respect to number of messages and $20 \%$ with respect to number of unique MMSI detected was achieved using the upgraded algorithm compared with the original for AISSat-1 [23]. The results presented in this paper representing the AISSat satellites are all from using the upgraded algorithm.

A third AISSat satellite, AISSat-3, was lost in a launch failure in 2017. The AISSat-3 satellite was practically identical to the AISSat-1 and -2, but it was equipped with an ASR300 AIS payload. The ASR300 is of the same form factor as ASR100, but has a larger FPGA with the capacity for more advanced algorithms than ASR100 as well as the ability to operate on all four AIS channels simultaneously. While no in-orbit results from ASR300 on-board AISSat-3 are available, FFI operate an AIS payload, NORAIS2, on the International Space Station that is in essence an ASR300 receiver. The evaluation of the ship tracking capability of the NORAIS2 receiver is beyond the scope of this paper, but a significant performance improvement over the ASR100 is achieved.

The NorSat-1 and -2 satellites, launched simultaneously in July 2017 into $\sim 98^{\circ}$ inclination, $\sim 600-\mathrm{km}$ altitude orbits, are larger than the AISSat satellites, with an approximate $20 \times 30 \times 40-\mathrm{cm}^{3}$ satellite body in addition to fixed solar panel "wings" that extend out from the body, weighing around $16 \mathrm{~kg}[4,24,25]$. Both NorSat satellites have multiple payloads, but the main payload is the ASRx50 AIS 
payload and the only payload of importance to this paper. The ASRx50 is yet an improvement on the ASR300 AIS payload with even more advanced decoding algorithms, supporting simultaneous demodulation on six channels, all subdividing the channels into multiple offset frequency zonals, a concept similar to that described by [5], developed by Kongsberg Seatex under an ESA contract [26]. ASRx50, furthermore, has a large memory capacity for sampling operations in addition to supporting four antennas and providing dual redundancy. Compared with the previous ASR 100 and ASR300 generation, the noise from the electronics in the AIS frequency band has also been further reduced.

Furthermore, NorSat-1 is equipped with two low-gain monopole antennas mounted perpendicular to each other for antenna diversity. NorSat-2 is also equipped with two antennas, a monopole and a high-gain Yagi-Uda antenna, the latter primarily for use by the other NorSat-2 payload [24]. An RF switch also connects the Yagi-Uda antenna to the AIS payload, however, but the duty cycle is shared between the two payloads [27].

\section{Methodology}

A general method to estimate the ship tracking capability of space-based AIS systems developed at FFI [28] has been used for the results presented in this paper, though other methods exist [29]. The results are based only on the data recorded by the space-based AIS systems themselves and has been argued to result in an upper bound on a space-based AIS system performance for moving ships on the world's oceans. The method can be used to quantify the ship tracking capability of any space-based AIS system, with the only metadata required being an additional timestamp upon reception of the AIS message. It is important to note that the ship tracking capability is not an AIS receiver performance measure (defined by a message detection probability by way of bit error rates vs. signal-to-noise ratios), but rather a space-based AIS system performance measure (defined by the capability of re-detecting ships as they move around the globe). Electromagnetic interference from the satellite platform and/or terrestrial sources can reduce the AIS receiver performance compared to that achieved in benchmarking on ground in ideal or constructed conditions. In-orbit operations of the satellite and/or ground segment may also reduce the raw performance of the AIS receiver and space system by only downloading a single message per MMSI per timeframe, or reducing the duty cycle for power management reasons, sharing antenna access with other satellites, or performing non-optimal attitude manoeuvres, etc. Since the ship tracking capability algorithm uses in-orbit data only, all these degradation effects are captured to make a total system ship tracking capability performance metric. For operational users, yet another parameter should likely be quantified for a total quality of service metric, namely the data latency [30], but that is considered beyond the scope of this paper.

The basic output of the ship tracking capability algorithm is the probability of re-detecting an already detected ship; the next time, the space-based AIS system covers the area in which the ship is, or more succinctly, a first access update probability. To yield a 12 or $24 \mathrm{~h}$ update probability, the first access update probability can be statistically accumulated, taking into account the orbital parameters of the space-based AIS system. Statistical accumulation, however, assumes that there is an equal chance of detecting all ships, but, in reality, some ships are harder to detect because of poor AIS equipment installation or configuration, or the ships are at rest, transmitting a few AIS messages for the space-based AIS system to receive, for instance. Thus, the final update probability is generally overestimated when statistically accumulating the first access update probability. For all caveats of the algorithm, the reader is referred to the original paper [28].

\subsection{Removing re-used MMSI numbers and MMSI numbers reporting erroneous positions}

In-orbit experience has shown that many MMSI numbers (MMSIs) are used by more than one ship. To calculate a ship tracking capability per MMSI, re-used MMSIs must be removed, for instance with method used in [28]. Furthermore, MMSIs reporting erroneous positions in the AIS messages are removed. Erroneous positions are defined as positions outside the field of view. Messages reporting the default position $181^{\circ} \mathrm{W}, 91^{\circ} \mathrm{N}$ are not used by the algorithm, such that no MMSI will be removed for having reported $181^{\circ} \mathrm{W}, 91^{\circ} \mathrm{N}$ during the analysis timeframe. The exception is if $181^{\circ} \mathrm{W}, 91^{\circ} \mathrm{N}$ is the only position the MMSI has reported during the analysis timeframe in which case it is difficult to track its movements. The remaining MMSIs, not being re-used or reporting erroneous positions are then tracked over the analysis timeframe. As reported in [28], fewer than 2\% of MMSIs are excluded based on the rules described. For transparency, the number of excluded vessels is presented for all results in Sects. 3 and 4.

\section{NorSat ship tracking capability}

For the NorSat-1 and NorSat- 2 results presented, the data set was gathered over a 26-day period from 1st January to 26th January 2018. During this period, the satellites had drifted apart in their orbit, such that NorSat- 2 trailed NorSat- 1 by approximately $1 \mathrm{~h}$, and because of the ship movements and earth rotation during this time, the satellites do not see exactly the same maritime picture during their orbits. 
For a single satellite ship trakcing capability, the results from NorSat-1 are presented as representative for the AIS receiver technology on both NorSat-1 and NorSat- 2 in order to reduce the number of similar plots. NorSat-1 results are furthermore most representative to use in comparison with the first generation Norwegian AIS satellites and technology presented in Sect. 4 since both NorSat-1 antennas are monopoles like the single antenna on the AISSat satellites. The ship tracking capability for both satellites has been calculated and the results confirm very smilar performance visually in spite of all the small differences in coverage, antenna configuration, and duty cycles between the two satellites.

\subsection{NorSat-1 ship tracking capability}

The ship tracking capability of NorSat-1 is presented in Fig. 2 for operation on the AIS1 and AIS2 channels in the upper plot, and for operation on AIS3 and AIS4 in the lower plot. Over the 26-day ship tracking period, the number of vessels detected, those excluded (according to the rules of the algorithm, ref. Sect. 2), those only detected once, and the resulting number of vessels tracked are shown in Table 1.

Statistically accumulating the results presented in Fig. 2 over $12 \mathrm{~h}$ worth of orbits yields the ship tracking capability presented in Fig. 3.

The results of Figs. 2 and 3 clearly demonstrate the improved ship tracking capability offered by the frequency

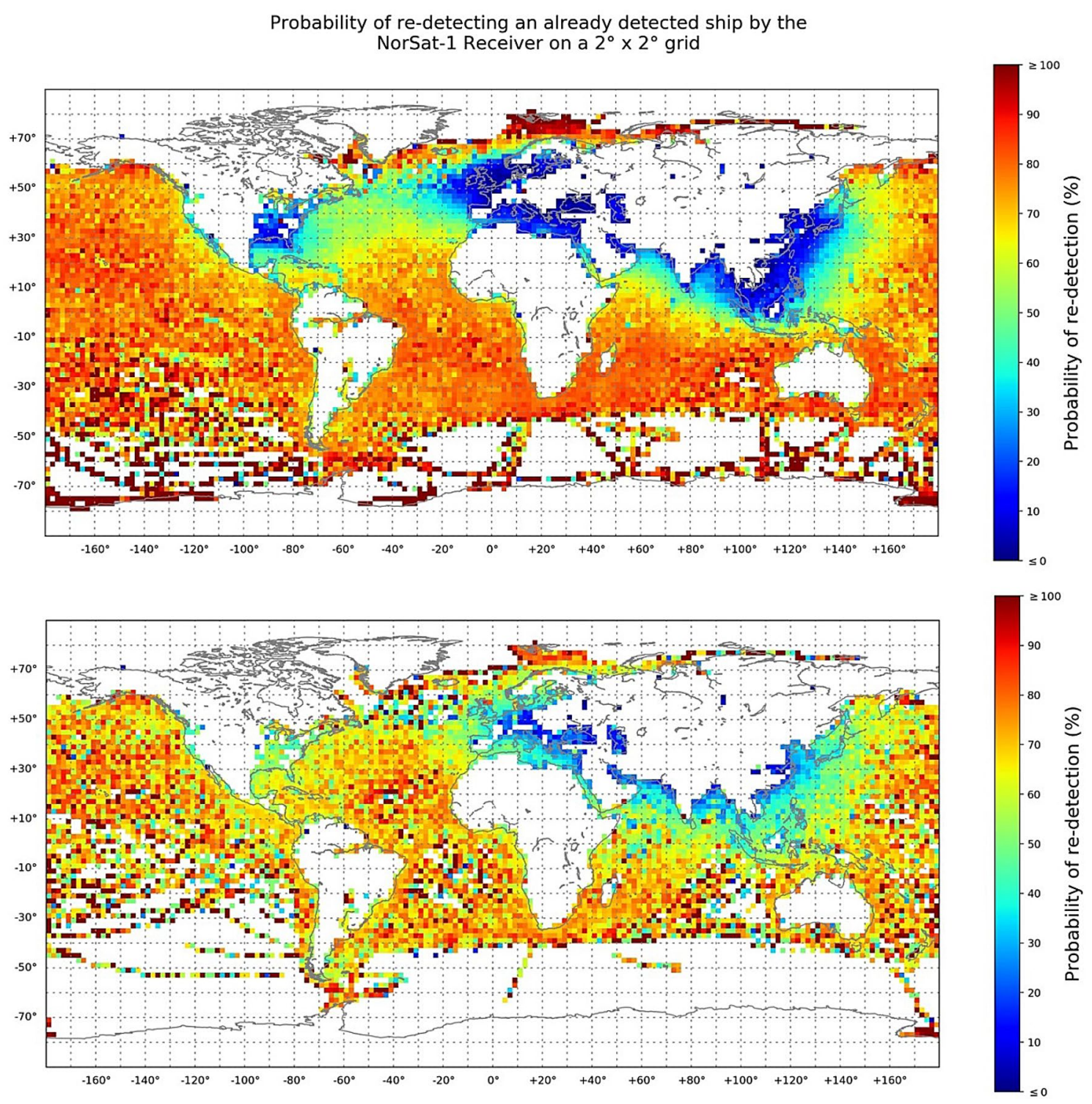

Fig. 2 NorSat-1 first access update probability using the AIS1 and AIS2 (top) or AIS3 and AIS4 (bottom) channels. The number of vessels in the plots is detailed in Table 1 
Table 1 NorSat-1 ship tracking algorithm statistics

\begin{tabular}{lllll}
\hline NorSat-1 configuration & Detected & Excluded & $\begin{array}{l}\text { Only } \\
\text { detected } \\
\text { once }\end{array}$ & Tracked \\
\hline AIS1 and AIS2 & 63,446 & 1187 & 3625 & 58,634 \\
AIS3 and AIS4 & 17,280 & 134 & 334 & 16,812 \\
\hline
\end{tabular}

diversity with the dedicated long-range message transmitted on the AIS3 and AIS4 channels. The first access update probability in high-vessel-density areas such as the North Sea, English Channel, Mediterranean, East and South China Sea, and the Gulf of Mexico has increased by around $20 \%$ points. The worst case $12 \mathrm{~h}$ update probability on the world's oceans is seen to be near 70\%. Closer to the coast and inland, the performance drops, however.

\subsection{NorSat-1 and NorSat-2 combined ship tracking capability}

The $12 \mathrm{~h}$ statistically accumulated ship tracking capability of the NorSat-1 and NorSat-2 combined is presented in Fig. 4 for operation on the AIS1 and AIS2 channels in the upper plot, and for operation on AIS3 and AIS4 in the lower plot. Over the 26-day ship tracking period, the number of vessels detected, those excluded (according to the rules of the ship

Average probability of re-detecting an already detected ship by the NorSat-1 Receiver on a $2^{\circ} \times 2^{\circ}$ grid within 12 hours
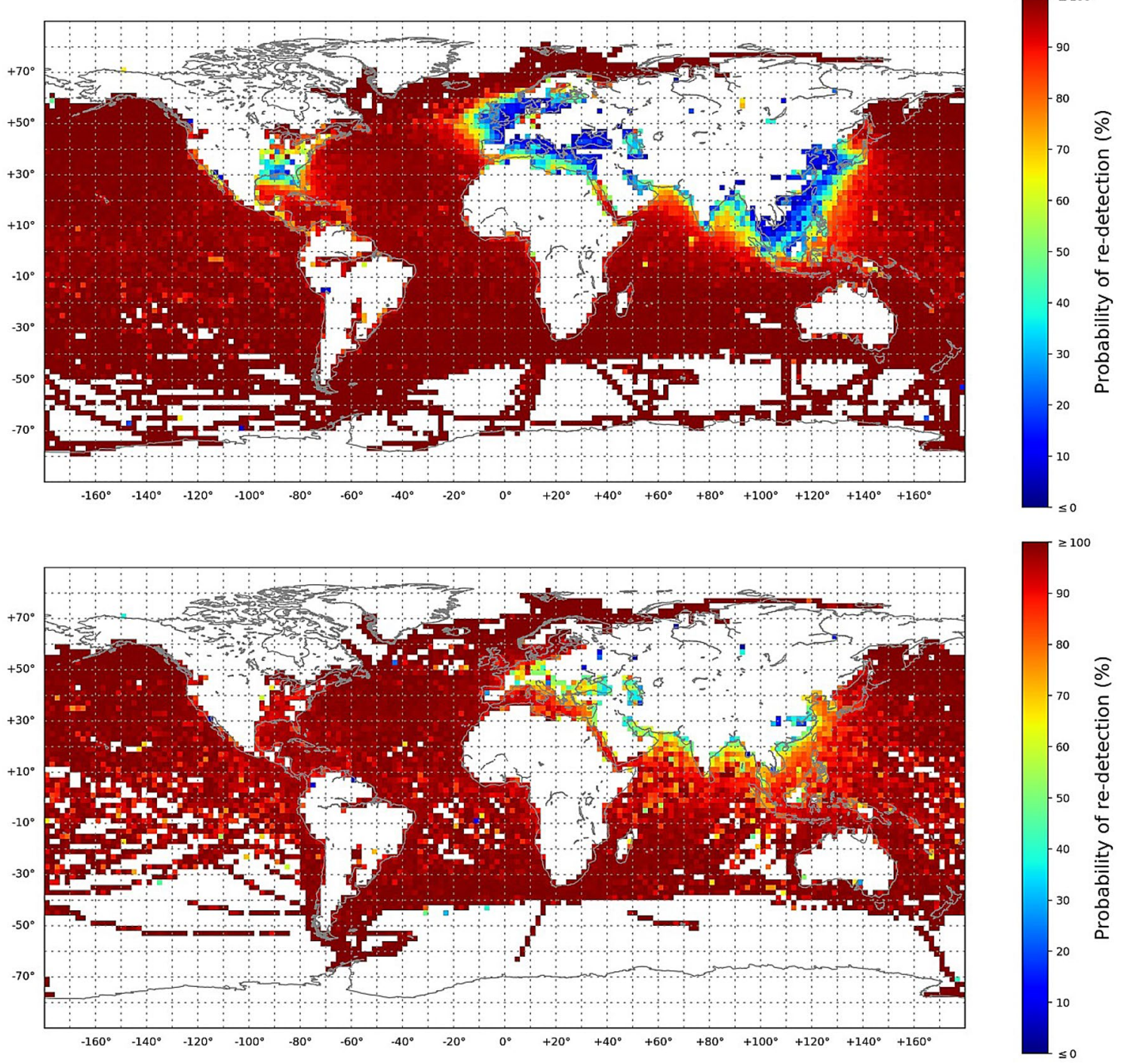

Fig. 3 NorSat-1 $12 \mathrm{~h}$ update probability using the AIS1 and AIS2 (top) or AIS3 and AIS4 (bottom) channels. The number of vessels in the plots is detailed in Table 1 
Average probability of re-detecting an already detected ship by the NorSat- 1 and NorSat-2 Receiver on a $2^{\circ} \times 2^{\circ}$ grid within 12 hours
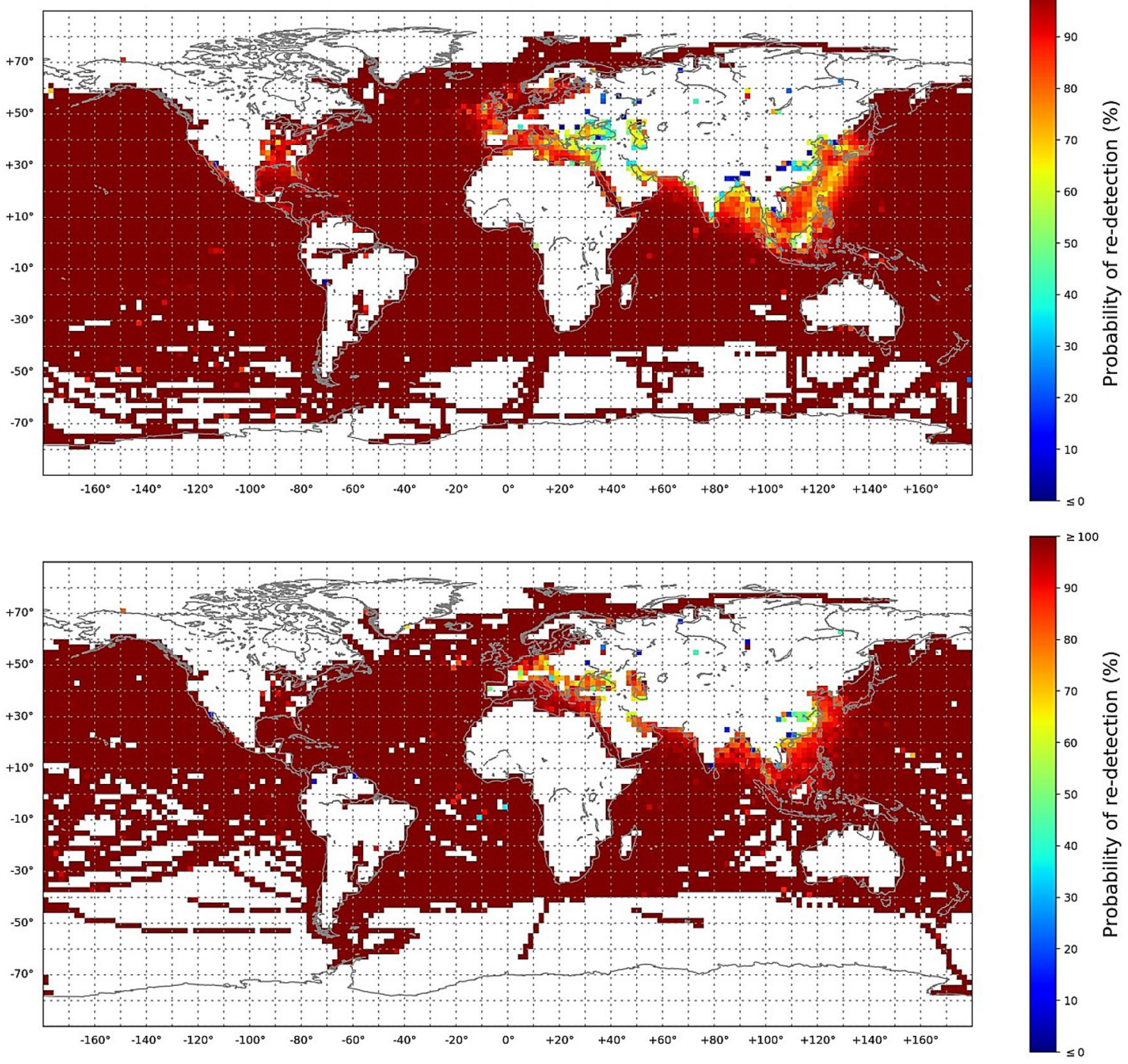

Fig. 4 Combined NorSat- 1 and NorSat-2 $12 \mathrm{~h}$ update probability using the AIS1 and AIS2 (top) or AIS3 and AIS4 (bottom) channels. The number of vessels in the plots is detailed in Table 2

Table 2 NorSat- 1 and NorSat-2 ship tracking algorithm statistics

\begin{tabular}{|c|c|c|c|c|}
\hline Satellite system and configuration & Detected & excluded & $\begin{array}{l}\text { Only } \\
\text { detected } \\
\text { once }\end{array}$ & Tracked \\
\hline NorSat-1, AIS1 and AIS2 & 63,446 & 1187 & 3625 & 58,634 \\
\hline NorSat-2, AIS1 and AIS2 & 64,309 & 1650 & 1540 & 61,119 \\
\hline NorSat- 1 and NorSat- 2 combined, AIS 1 and AIS 2 & 68,161 & 2103 & 4932 & 61,126 \\
\hline NorSat-1, AIS3 and AIS4 & 17,280 & 134 & 334 & 16,812 \\
\hline NorSat-2, AIS4 and AIS4 & 17,177 & 176 & 140 & 16,861 \\
\hline NorSat-1 and NorSat-2 combined, AIS3 and AIS4 & 17,531 & 250 & 361 & 16,920 \\
\hline
\end{tabular}


Probability of re-detecting an already detected ship by the AISSat-2 Receiver on a $2^{\circ} \times 2^{\circ}$ grid

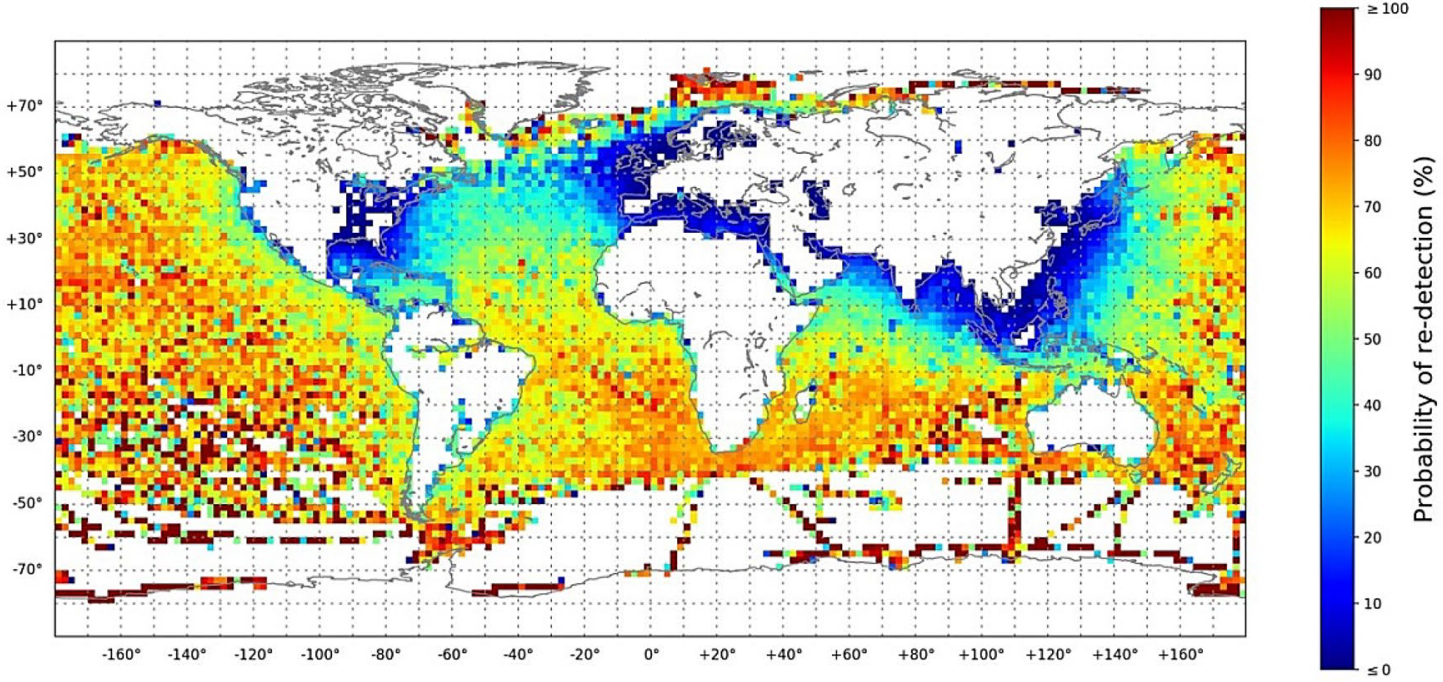

Probability of re-detecting an already detected ship by the NorSat-1 Receiver on a $2^{\circ} \times 2^{\circ}$ grid
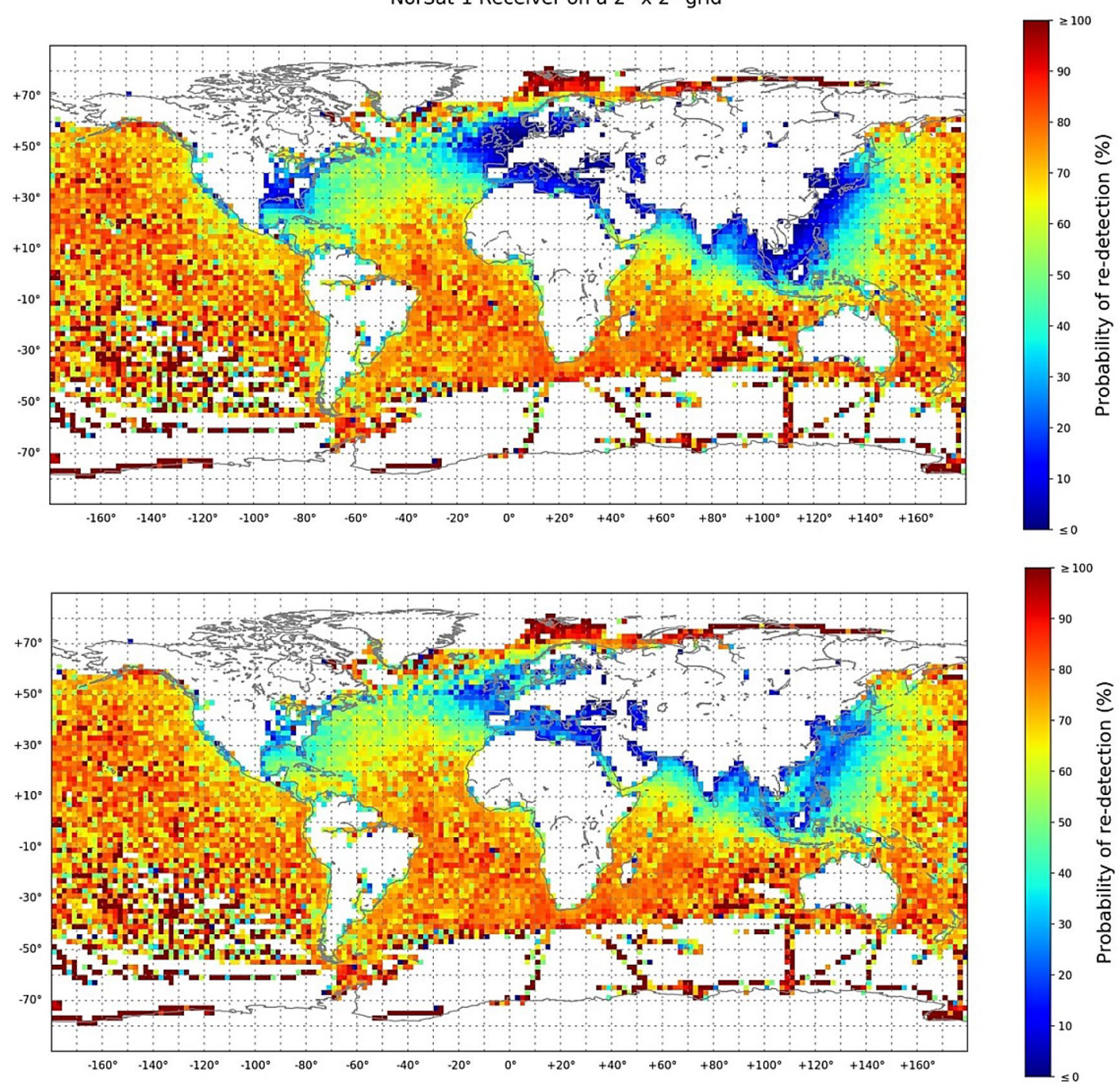
४Fig. 5 First access update probability by AISSat-2 (top), NorSat-1 operating on AIS1 and AIS2 only (middle), and NorSat-1 operating on all four AIS channels (bottom). The number of vessels in the plots is detailed in Table 3

tracking algorithm), those only detected once, and the resulting number of vessels tracked are shown in Table 2.

Evident from the summary results in Table 2 is that the number of vessels excluded and only detected once is lower for the combination of NorSat- 1 and NorSat- 2 compared with the sum of the respective numbers for NorSat-1 and -2 individually. As previously mentioned, NorSat-2 trailed NorSat-1 by approximately $1 \mathrm{~h}$, such that differences could be related to ship movements and earth rotation during this gap. However, the results may also indicate that the combination of a low-gain antenna combined with a higher gain antenna, like the monopole and Yagi-Uda antenna combination used by NorSat-2, could improve the ship tracking capability. Unfortunately for the research in this paper, the high-gain antenna of NorSat-2 is a shared antenna between the AIS payload and another payload with the other payload operations being the driver for the high-gain antenna pointing, as described previously in Sect. 1.2. In other words, the Yagi-Uda antenna is not used or pointed to intentionally optimise AIS performance, but this could be a topic for future experimentation and research.

\section{Ship tracking capability improvements}

In this section, the ship tracking capability of AISSat-2, as a representative of the first-generation technology, is compared with NorSat-1, representing the current state-of-theart technology. The state-of-the-art technology includes advances in decoding algorithms, antenna diversity in addition to frequency diversity by way of a four-channel AIS receiver taking advantage of the updated AIS system standard with a dedicated long-range message on the AIS3 and AIS4 channels. A 15-day ship tracking period from 1st January 00:00 UTC to 14th January 21:00 UTC 2018 is used where both AISSat- 2 and NorSat-1 were operated with a high system uptime for the fairest comparison possible.

The first comparison of the ship tracking capability of AISSat-2 and NorSat-1 is presented in Fig. 5. Only MMSIs that are in both data sets are used for a representative comparison of the calculated ship tracking capability. The top plot shows the AISSat-2 capability, while the middle plot shows NorSat-1 operating only on AIS 1 and AIS2 like AISSat- 2 does, and shows the improvements realised by advances in algorithms and antenna diversity. The lower plot shows NorSat-1 operating on all channels, but still only ship tracking MMSI numbers also detected by AISSat-2, thus including the performance improvements realised by frequency diversity and the use of the longrange AIS message. Statistically accumulating the results presented in Fig. 5 over $12 \mathrm{~h}$ worth of orbits yields the ship tracking capability presented in Fig. 6.

Over the 15-day ship tracking period, the number of vessels detected, those excluded (according to the rules of the algorithm), those only detected once, and the resulting number of vessels tracked is shown in Table 3.

In general, NorSat-1 detects more MMSI numbers per timeframe than AISSat-2, even when considering AIS 1 and AIS2 results only, and the improvement can be seen in all the areas where space-based AIS typically struggles like the Mediterranean, South China Sea, North Indian Ocean, Gulf of Mexico, and the Great lakes, especially after $12 \mathrm{~h}$. In addition, many more MMSI numbers are only seen once by AISSat- 2 compared with NorSat- 1 in the 15-day timeframe. As such, the improvement from AISSat- 2 to NorSat- 1 can actually be considered even greater than the ship tracking capability results show, since NorSat-1 technology detects more vessels in any case. Quantified, NorSat-1 detected 20\% more vessels on the AIS1 and AIS2 channels, and 34\% more vessels in total compared with AISSat- 2 over the same timeframe. For the ship tracking capability, AISSat-2 could not track 13\% of the commonly detected vessels, since they were only detected once, while only $2 \%$ of the commonly detected vessels could not be tracked by NorSat-1 due to only being detected once. Comparing the first access update probability of AISSat- 2 and NorSat-1 operating on the AIS 1 and AIS2 channels only, up to a $20 \%$ point increase can easily be seen in large parts of the Atlantic, Indian Ocean, and Pacific.

Specifically for the main area of interest for the Norwegian satellites, the performance in the Norwegian and Baltic Sea has also improved significantly, as shown in Fig. 7, for AISSat-2 (left column) and NorSat-1 (right column) for the first pass re-detection and after $12 \mathrm{~h}$ ship tracking capability.

The second performance comparison made to highlight the improved ship tracking capability of the state-of-the-art technology once again uses MMSI numbers detected both by AISSat- 2 and NorSat-1, but this time only those detected by NorSat- 1 operations on the AIS3 and AIS4 channels. The results, presented in Fig. 8, showcase future potential capability of the state-of-the-art technology as more and more vessels use the AIS3 and AIS4 channels and the dedicated long-range AIS message. The top plot shows the AISSat-2 capability, while lower plot shows the NorSat- 1 capability. Statistically accumulating the results presented in Fig. 8 over $12 \mathrm{~h}$ worth of orbits yield the ship tracking capability presented in Fig. 9.

Over the 15-day ship tracking period, the number of vessels detected, those excluded (according to the rules of the algorithm), those only detected once, and the resulting 
Average probability of re-detecting an already detected ship by the AISSat- 2 Receiver on a $2^{\circ} \times 2^{\circ}$ grid within 12 hours

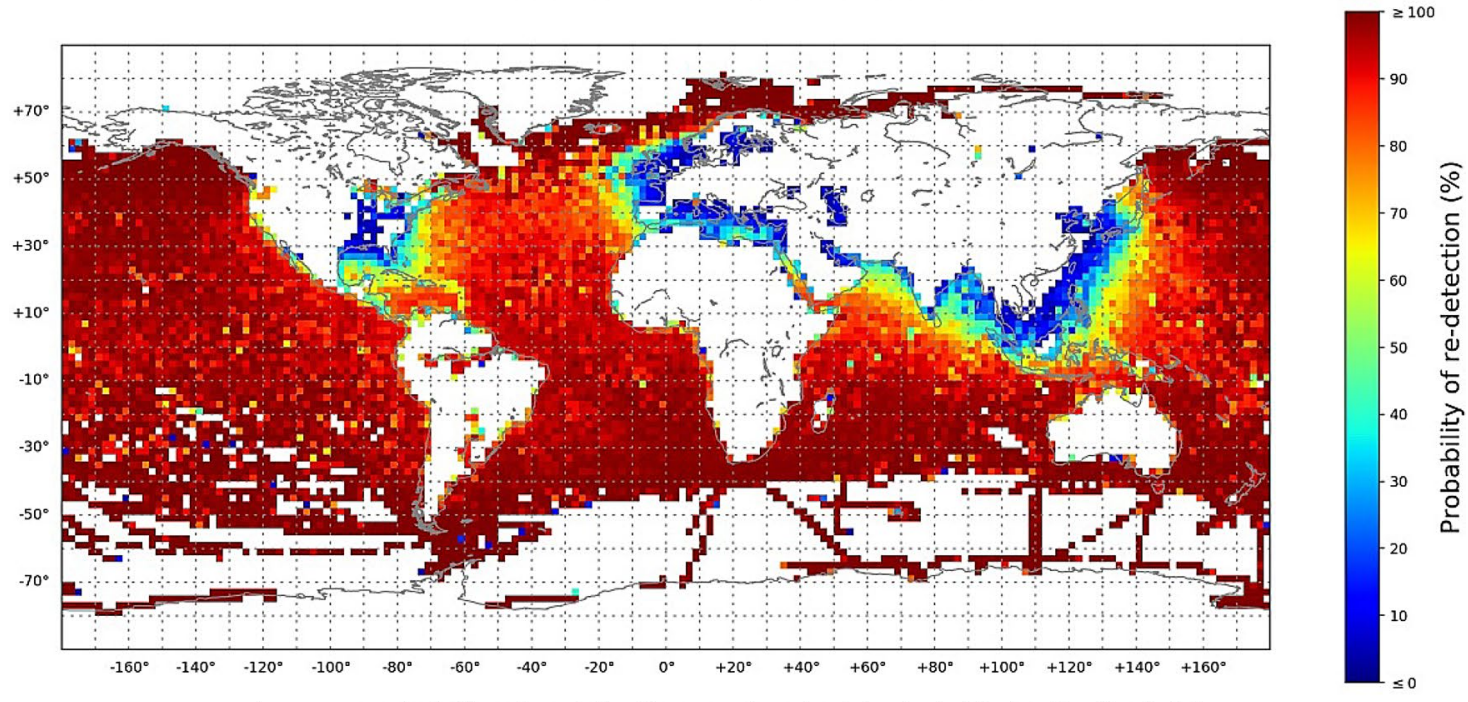

Average probability of re-detecting an already detected ship by the NorSat- 1
Receiver on $a 2^{\circ} \times 2^{\circ}$ grid within 12 hours
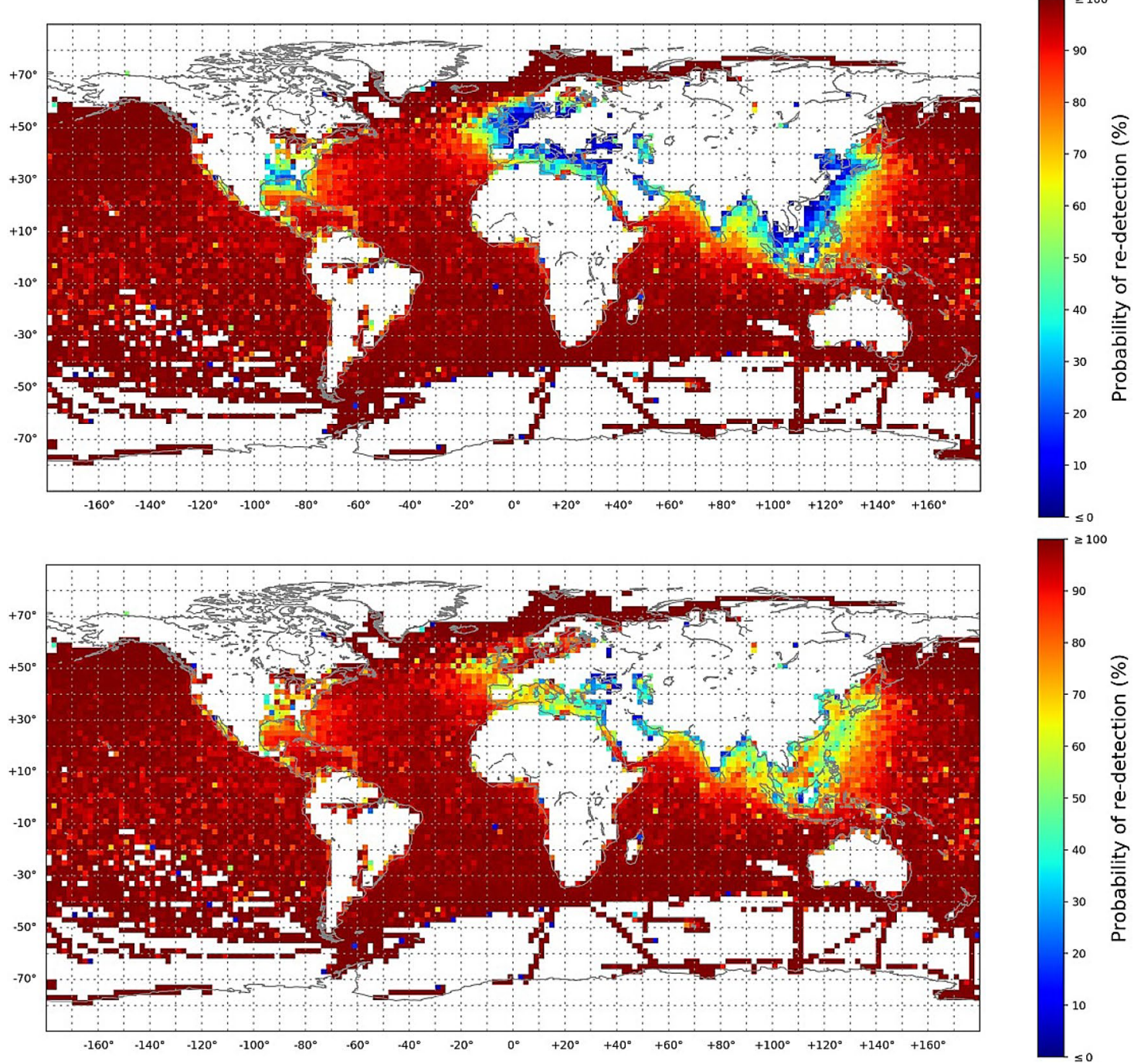
४Fig. 612 hours update probability by AISSat-2 (top), NorSat-1 operating on AIS1 and AIS2 only (middle), and NorSat-1 operating on all four AIS channels (bottom). The number of vessels in the plots is detailed in Table 3

number of vessels tracked are shown in Table 4. Note that more than half of the total number of MMSI detected by NorSat- 1 on the AIS3 and AIS4 channels is not detected by AISSat-2.

We observed that the AISSat-2 ship tracking performance for vessels that also operate on the AIS3 and AIS4 channels is seemingly better than the general AISSat-2 ship tracking performance previously presented in Figs. 5 and 6. Comparing the performance in the Atlantic Ocean, Bay of Bengal, and the Pacific Ocean east/southeast of Japan across to North and Central America, for instance, it seems that many of the vessels that have upgraded their installations to also operate on AIS3 and AIS4 are easier to detect (more dark red grid cells). The results could indicate that vessels that have upgraded their systems early have better installations, in general (possibly due to the servicing required to update/ upgrade the equipment), but detailed study of the typical signal strengths from these vessels has not been performed.

\section{Discussion}

The results presented in Sect. 3 show that, even with the improved algorithms and multiple antennas of the NorSat satellites, the AIS1 and AIS2 first access update probability, Fig. 2 top plot, is low in the high-vessel-density areas of the Mediterranean, North Sea, English Channel with surrounding waters and East and South China Sea. Looking at the frequency diversity offered by the AIS3 and AIS4 channels in the bottom plot of Fig. 2, with the dedicated long-range message, the performance in the aforementioned areas improves drastically, especially when statistically accumulating the results over $12 \mathrm{~h}$ as shown in bottom plots of Fig. 3. It is important to realise that it is the combination of a purpose made long-range message and dedicated frequencies that realise the performance gain, not one or the other alone. The use of the long-range message on the existing AIS 1 and AIS2 channels would not have been effective due to the existing co-channel interference. Likewise, using the AIS 1 and AIS2 channel AIS message set and rules on additional frequencies would not yield the performance gain seen either, again due to the co-channel interference that would occur.

In the Barents Sea and the northern Norwegian Sea, the original area of interest for the AISSat-1 mission, the ship tracking capability is actually better on the AIS1 and AIS2 channels than on the AIS3 and AIS4 channels. The AIS3 and AIS4 channel performance rapidly becomes better further south in the Norwegian Sea, from the Norwegian mainland and further south, evident from comparing the two plots in Fig. 2. As long as the total number of vessels is low, like in the Barents Sea and northern Norwegian Sea, especially in January, the higher AIS message transmission rate on the AIS1 and AIS2 channels aids the first access update probability due to the large number of messages per vessel available for the space-based AIS system to detect. On the AIS3 and AIS4 channels, only a few messages are transmitted per vessel per observation time, which is helpful in areas with much co-channel interference, but makes the system vulnerable to signal propagation effects.

Adding a second NorSat satellite and statistically accumulating the results over $12 \mathrm{~h}$ as shown in Fig. 4 yield very impressive results. A typical $12 \mathrm{~h}$ update probability approaching $90 \%$ in most of the high-vessel-density areas even on the AIS1 and AIS2 channels is achieved by the NorSat-1 and NorSat-2 satellites combined. Still, comparing the results of the single NorSat-1 operating on AIS3 and AIS4 channels only, bottom plot of Fig. 3, with the combined capability of NorSat- 1 and NorSat- 2 operating on AIS1 and AIS2 only, top plot in Fig. 4, indicates that one satellite with advanced algorithms, multiple antennas, and AIS3 and AIS4 channel decoding capability exceeds the performance of two satellites operating only on the AIS1 and AIS2 channels, even with advanced algorithms and multiple antennas in the high-vessel-density areas.

Furthermore, the results in Sect. 4, where the state-ofthe-art technology, represented by NorSat-1, was compared with the first-generation technology, represented by AISSat-2, also indicate that the greatest improvement in ship tracking capability in the high-vessel-density areas is achieved by a four-channel receiver. However, the use of the AIS3 and AIS4 channels is still not widespread, with only 17,531 out of at least 68,161 vessels detected using the AIS3 and AIS4 channels versus the AIS1 and AIS2 channels, as shown in Table 2. In 2014, the number of AIS3 and AIS4 reporting vessels was estimated to less than 2300 using an ASR 100 type receiver on the International Space Station $[22,28]$. With a linear adoption rate, the full fleet adoption of the AIS3 and AIS4 channels is not expected for another 14 years.

Considering the still low use of the AIS3 and AIS4 channels, the results in Sect. 4 also indicate that, in the short term, the total fleet ship tracking capability is most improved by upgrading to more advanced algorithms and adding antenna diversity. Especially in the Norwegian Sea, the results shown in Fig. 7 show that the state-of-the-art technology, operating only on AIS1 and AIS2, provides near $100 \%$ re-detection probability all the way south to the North Sea, while similar results were only achieved in the Barents Sea and northern Norwegian Sea for the previous generation technology. 
Table 3 AISSat-2 and NorSat-1 ship tracking algorithm statistics

\begin{tabular}{llllll}
\hline Satellite system and configuration & Detected & $\begin{array}{l}\text { Commonly } \\
\text { detected }\end{array}$ & $\begin{array}{l}\text { Commonly } \\
\text { excluded }\end{array}$ & $\begin{array}{l}\text { Only } \\
\text { detected } \\
\text { once }\end{array}$ & Tracked \\
\hline AISSat-2 & 47,725 & 42,248 & 1266 & 5776 & 36,472 \\
NorSat-1, AIS1 and AIS2 only & 57,554 & 42,248 & 1266 & 971 & 41,277 \\
NorSat-1, all channels & 63,956 & 42,815 & 1345 & 839 & 41,976 \\
\hline
\end{tabular}

Probability of re-detecting an already detected ship by the AISSat-2 Receiver on a $2^{\circ} \times 2^{\circ}$ grid
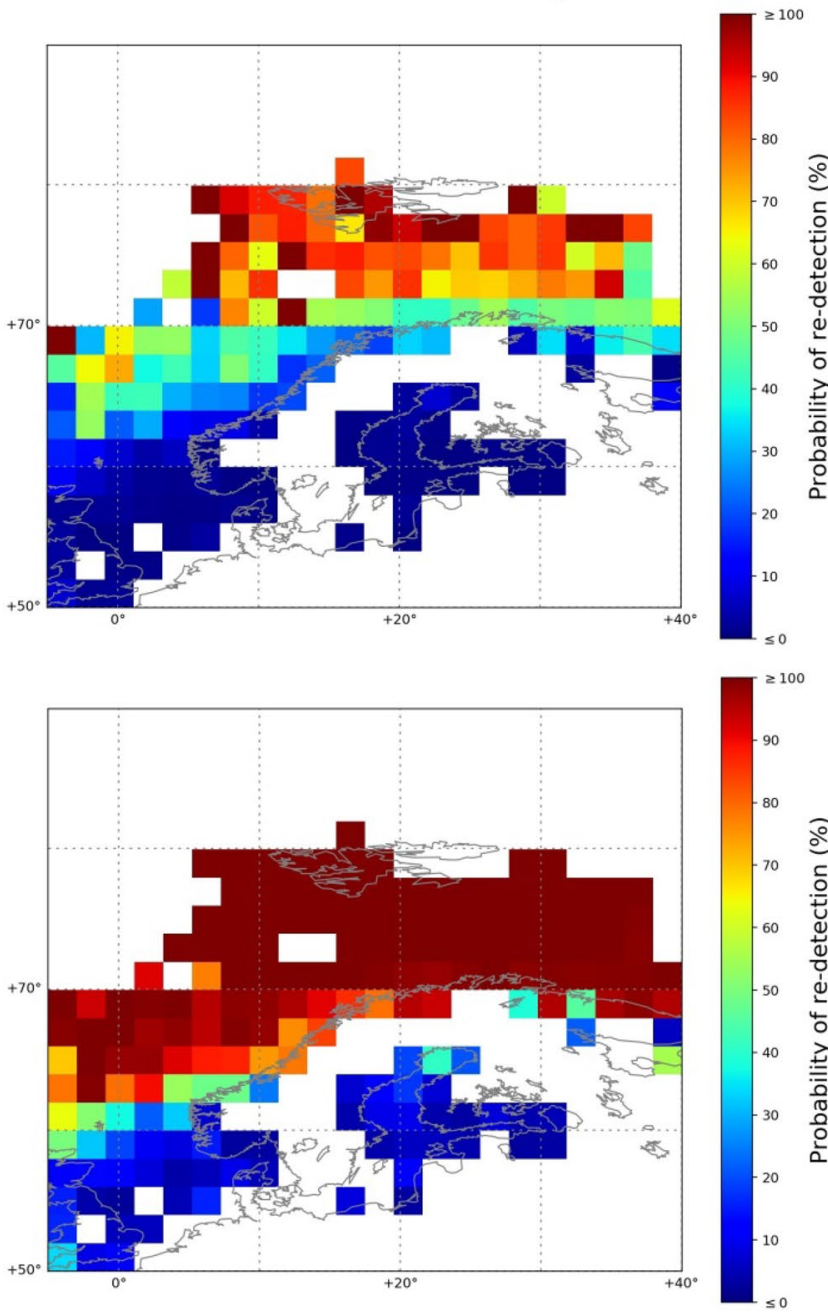

Probability of re-detecting an already detected ship by the NorSat-1 Receiver on a $2^{\circ} \times 2^{\circ}$ grid
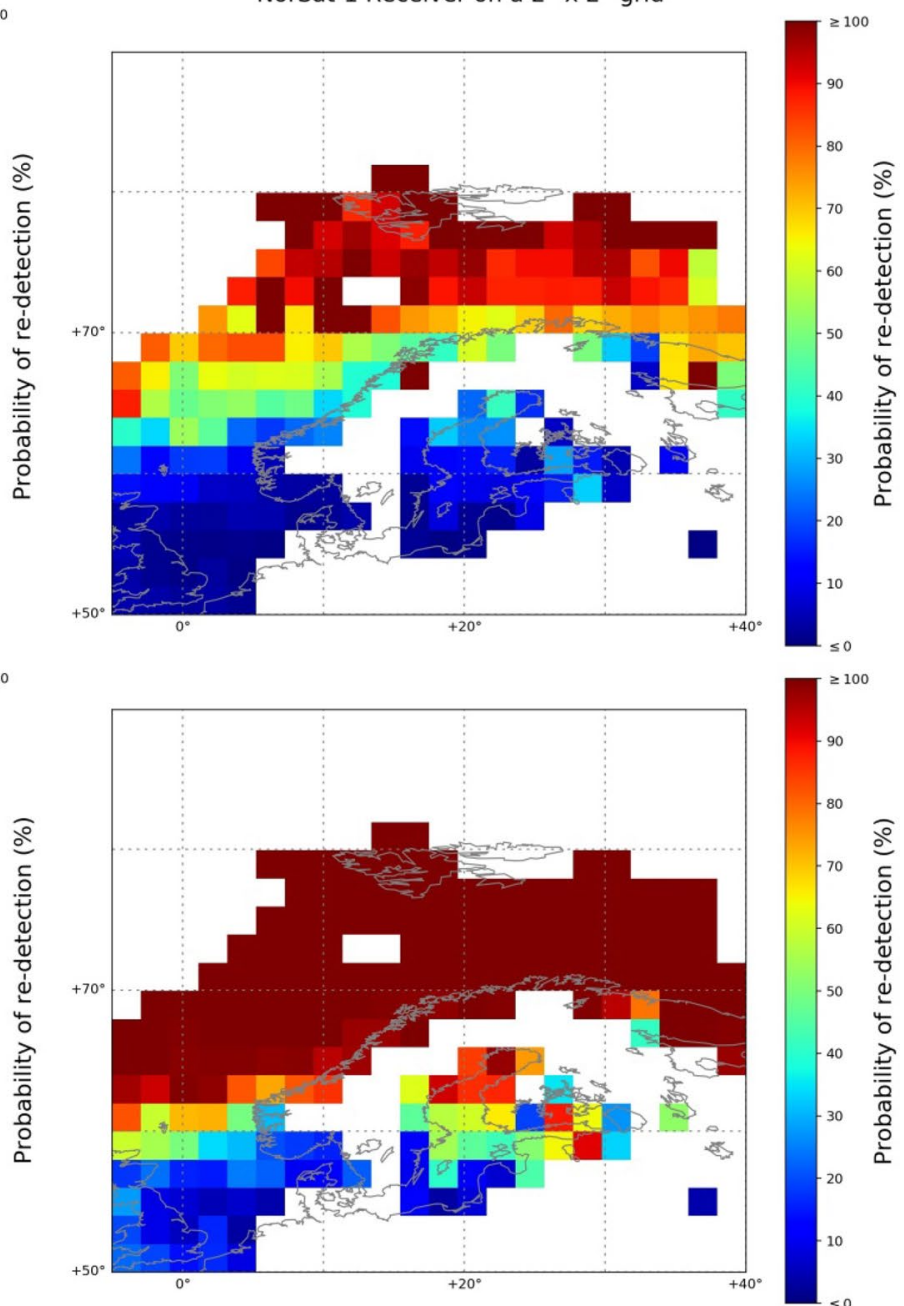

Fig. 7 First access update probability by AISSat-2 (top left) and NorSat-1 operating on AIS1 and AIS2 only (top right) and the statistically accumulated $12 \mathrm{~h}$ update probability by AISSat-2 (bottom left) and NorSat-1 (bottom right) operating on AIS1 and AIS2

In the medium term, when more vessels should be using the AIS3 and AIS4 channels, the results indicate that the most gain may be achieved using four-channel receivers. A framework for estimating the saturation point as a function of required detection probability, observation time, and reporting interval has been developed by Høye [31]. To maintain a $90 \%$ detection probability, the maximum number of ships within the field of view in an area is estimated to
11,000 , given a 3-min reporting interval over two channels as implemented by the long-range message on AIS3 and AIS4, and assuming 20-min total observation time. The total observation time will vary with antenna pattern and satellite orbital parameters, but, as an example, the total observation time achieved within $12 \mathrm{~h}$ as a function of latitude by satellites with omnidirectional, e.g., monopole, antennas at $600-\mathrm{km}$ altitude for two different orbital inclinations are 


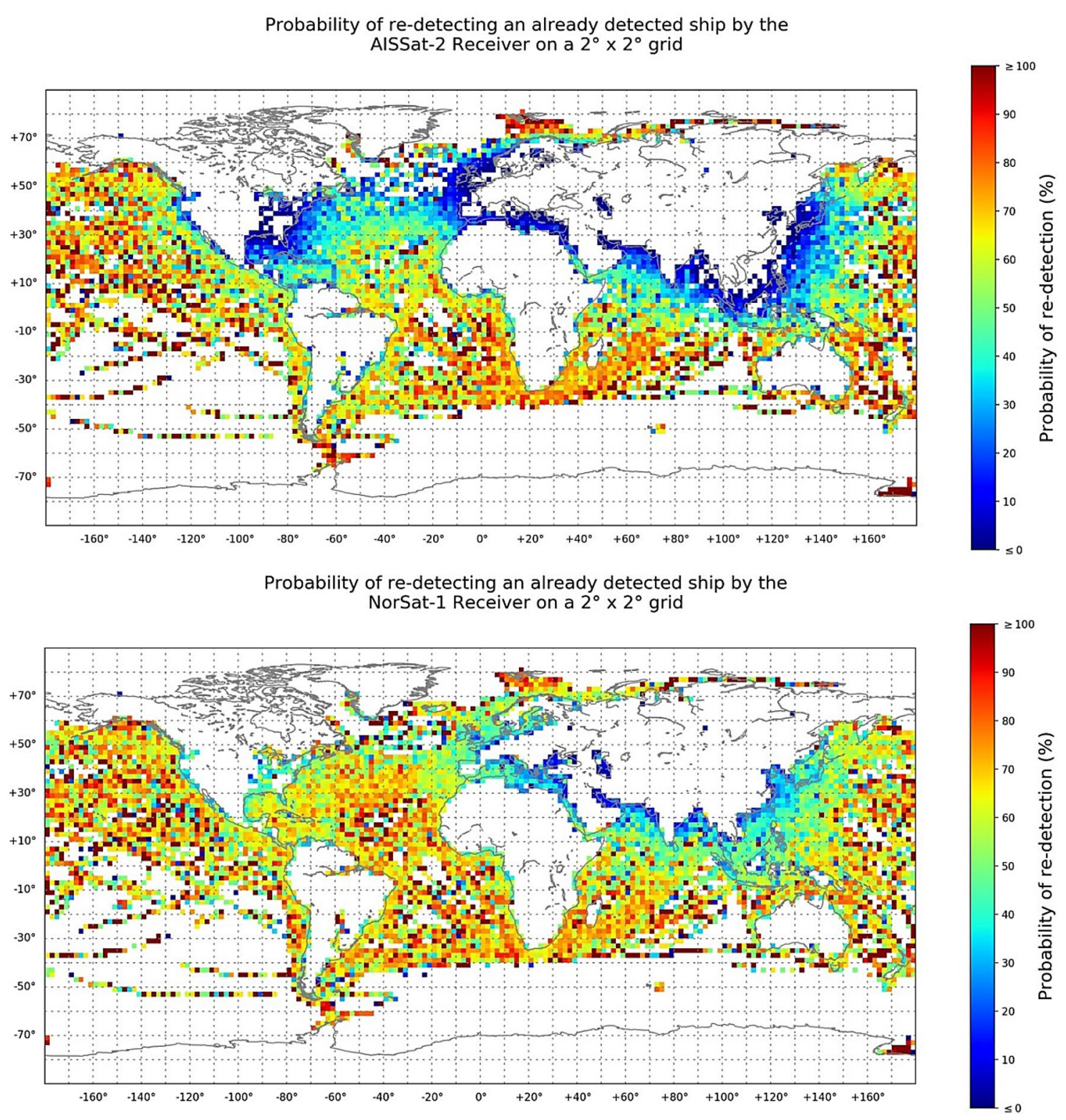

Fig. 8 First access update probability by AISSat-2 (top) and NorSat-1 operating on the AIS3 and AIS4 channels (bottom). The number of vessels in the plots is detailed in Table 4

presented in Table 5. The latitudes are chosen to cover the span of the high-vessel-density areas, from the southernmost parts of the South China Sea close to $0^{\circ} \mathrm{N}$, to the Mediterranean and East China Sea around $35^{\circ} \mathrm{N}$ to the North Sea around $55^{\circ} \mathrm{N}$.

Similar saturation numbers for the AIS1 and AIS2 channels are calculated to only 1750 vessels in the field of view simultaneously for $90 \%$ detection probability after 20 -min total observation time, approaching 2000 after 30 -min total observation time [31]. Olsen [32] estimated the number of vessels in the aforementioned high-vessel-density areas to be close to 10,000 active vessels per area at any given time by 2017 . Multiple high-vessel-density area and/or adjacent lower vessel-density areas can be within the field of view of a space-based AIS system simultaneously. While the saturation numbers are exceeded on the AIS1 and ASI2 channels for many space-based AIS systems' field of view, the results in the top plot of Fig. 4 show that the $12 \mathrm{~h}$ update probability in the high-vessel-density areas for state-of-the-art technology is generally exceeding $80 \%$. The total observation time can reach around 50 min after $12 \mathrm{~h}$ for NorSat-1 and NorSat-2 combined for the Mediterranean high-vessel-density area for example. Thus, the saturation point for AIS3 and AIS4 should not be exceeded even at full fleet adoption, given the current fleet size. 
Average probability of re-detecting an already detected ship by the AISSat- 2 Receiver on a $2^{\circ} \times 2^{\circ}$ grid within 12 hours

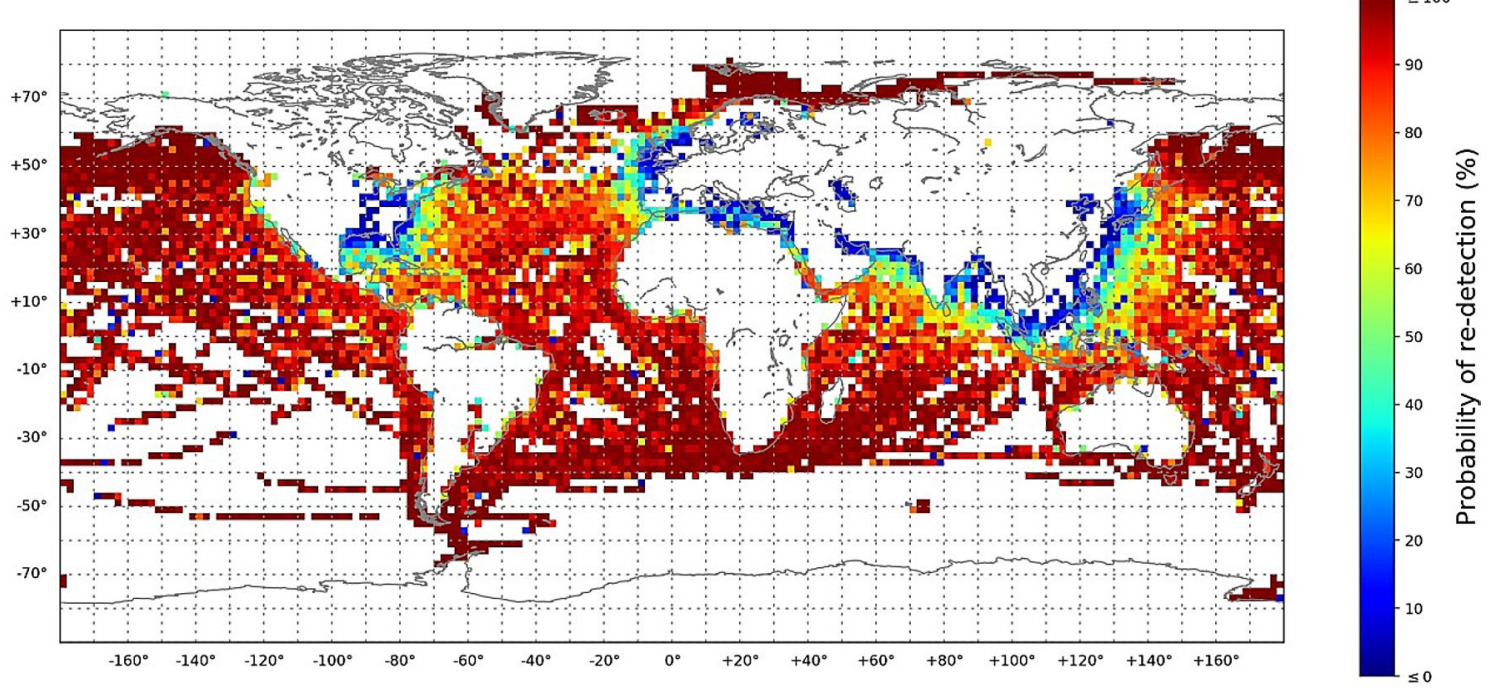

Average probability of re-detecting an already detected ship by the NorSat-1 Receiver on a $2^{\circ} \times 2^{\circ}$ grid within 12 hours

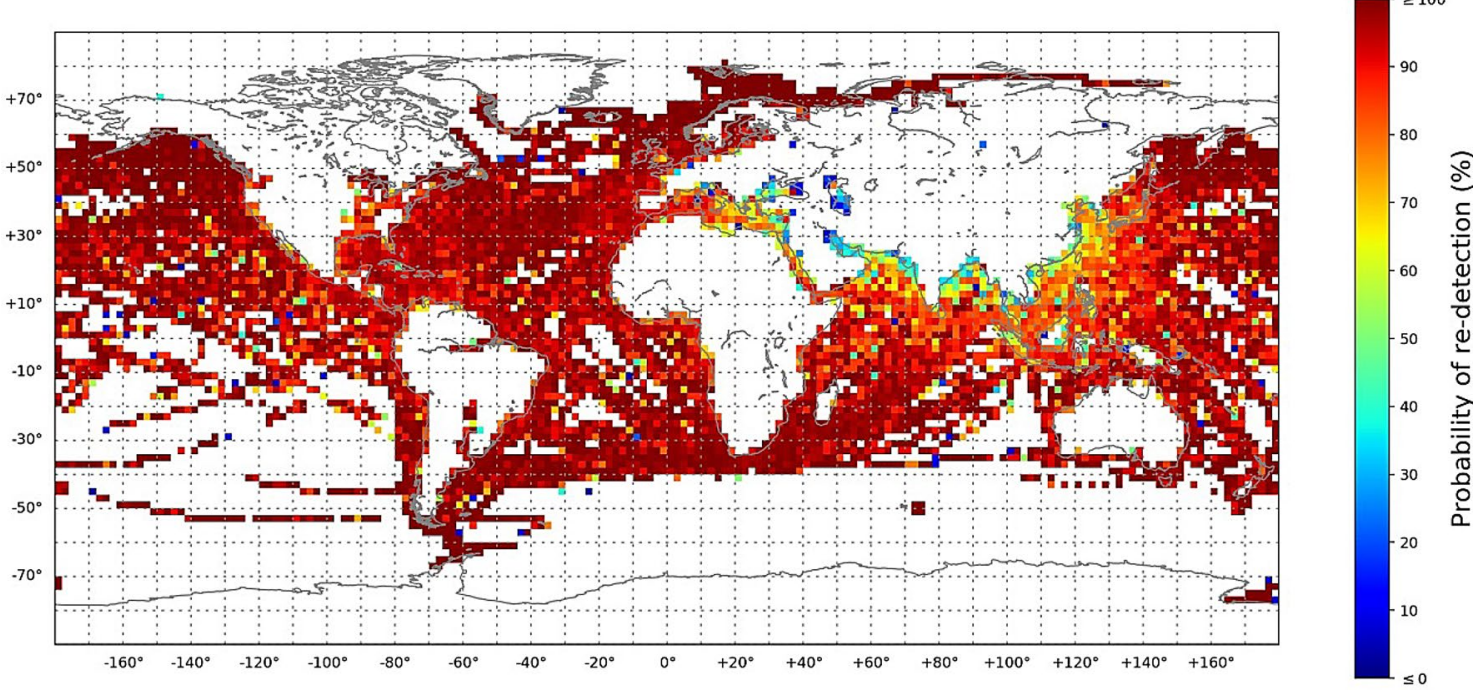

Fig. 912 hours update probability by AISSat-2 (top) and NorSat-1 operating on the AIS3 and AIS4 channels (bottom). The number of vessels in the plots is detailed in Table 4

Table 4 AISSat-2 and NorSat-1 ship tracking algorithm statistics

\begin{tabular}{llllll}
\hline Satellite system and configuration & Detected & $\begin{array}{l}\text { Commonly } \\
\text { detected }\end{array}$ & $\begin{array}{l}\text { Commonly } \\
\text { excluded }\end{array}$ & $\begin{array}{l}\text { Only } \\
\text { detected } \\
\text { once }\end{array}$ & Tracked \\
\hline AISSat-2 & N/A & 7397 & 79 & 992 & 6405 \\
NorSat-1, AIS3 and AIS4 only & 16,199 & 7397 & 79 & 23 & 7374 \\
\hline
\end{tabular}

Potential future work that was beyond the scope of this work is to investigate the use of one low-gain AIS antenna for maximum area coverage, and a second high-gain AIS antenna pointed to minimise the co-channel interference from high-vessel-density areas in an attempt to maximise the ship tracking capability in those difficult areas. The NorSat-2 satellite has the necessary hardware capable of supporting such experiments, but the shared access between multiple 
Table 5 Total observation time achieved within $12 \mathrm{~h}$ at different latitudes for a $600-\mathrm{km}$ altitude satellite with an omnidirectional antenna in two different orbital inclinations

Total observation time achieved within $12 \mathrm{~h}$ for a $600-\mathrm{km}$ altitude satellite with an omnidirectional antenna

\begin{tabular}{lll}
\hline Latitude $\left(^{\circ}\right)$ & \multicolumn{2}{l}{ Orbital inclination } \\
\cline { 2 - 3 } & $98^{\circ}(\mathrm{min})$ & $30^{\circ}(\mathrm{min})$ \\
\hline 0 & 20 & 43 \\
\pm 35 & 25 & 33 \\
\pm 55 & 40 & 0 \\
\hline
\end{tabular}

NorSat-2 payloads to the high-gain Yagi-Uda antenna could be prevent such experiments. Furthermore it could also be possible to isolate the effect of the antenna diversity somewhat by comparing the ship tracking capability of NorSat-1 using only one versus both antennas. Similarly it could be possible to isolate the effect of improved algorithms by comparing the ship tracking capability of NorSat-1 using a single antenna with that of an AISSat. While the full ship tracking capability of these configurations is beyond the scope of this work, a raw comparison of the number of MMSI detected for the aforementioned system configurations may give an indication about the improvement from antenna diversity and algorithms separately. NorSat-1 detected 52999 MMSI for the best case single antenna over the same timeframe as previously presented in Table 3 for AISSat- 2 and for NorSat-1 using both antennas. A raw comparison of the number of MMSI detected gives $11 \%$ increase in number of MMSI from algorithms alone (AISSat- 2 vs NorSat- 1 single antenna) and nearly $9 \%$ increase in number of MMSI from the antenna diversity (NorSat-1 single antenna vs NorSat-1 two antennas).

\section{Summary}

The ship tracking capability of the most recently launched Norwegian space-based AIS systems, NorSat-1 and NorSat-2, was quantified and shown to be significantly improved in all high-vessel-density areas compared with the first-generation AISSat-1 and AISSat-2 systems. Using NorSat-1 as a representative of state-of-the-art technology, in-orbit results quantified the improved global ship tracking capability gained by antenna diversity, frequency diversity and improved algorithms compared with the firstgeneration technology, represented by AISSat-2. While improved algorithms and antenna diversity have improved the capability significantly on their own, the frequency diversity introduced by the AIS3 and AIS4 channels and their use of only one purpose made long-range message, is the only viable option to enable a reliable ship tracking capability in the high-vessel-density areas for a single-satellite system. However, the AIS3 and AIS4 adoption level is still not sufficient to rely solely on frequency diversity as the only capability enhancer. Using multiple satellites with state-of-the-art technology, the ship tracking capability even when only operating on the AIS1 and AIS2 channels rival that of the AIS3 and AIS4 performance of a single-satellite system despite the significant co-channel interference present in the high-vessel-density areas.

In summary, NorSat-1 and NorSat-2 provide continuity to the Norwegian space-based AIS capability, with a ship tracking capability that significantly outperforms their AISSat-1 and AISSat-2 predecessors.

Open Access This article is distributed under the terms of the Creative Commons Attribution 4.0 International License (http://creativeco mmons.org/licenses/by/4.0/), which permits unrestricted use, distribution, and reproduction in any medium, provided you give appropriate credit to the original author(s) and the source, provide a link to the Creative Commons license, and indicate if changes were made.

\section{References}

1. Narheim, B.T., Helleren, Ø., Olsen, Ø., Olsen, R., Rosshaug, H., Beattie, A.M., Kekez, D.D., Zee, R.E.: AISSat-1 early results. In: Proceedings of the AIAA/USU Conference on Small Satellites, Reflections on the Past, SSC11-III-6 (2011). https://digit alcommons.usu.edu/smallsat/2011/all2011/26/. Accessed 5 Apr 2019

2. Helleren, Ø., Olsen, Ø., Narheim, B. T., Skauen, A. N., Olsen, R. B.: AISSat-1-2 years of service. In: Proceedings of the $4 S$ Symposium, Portorož, Slovenia (2012a)

3. Eriksen, T., Skauen, A., Narheim. B., Helleren, Ø., Olsen, Ø., Olsen, R.: Tracking Ship Traffic With Space-Based AIS: Experience Gained in First Months of Operations. In: Proceedings of the Waterside Security Conference, Marina di Carrara, Italy (2010). https://doi.org/10.1109/WSSC.2010.5730241

4. Harr, J., Jones, T., Andersen, B. N., Eriksen, T., Skauen, A. N., Svenes, K., Blindheim, E. V., Spydevold, I., Beattie, A., Bradbury, L. M., Cotten, B., Kekez. D., Mehradnia, P., Zee, R. E., Storesund, F.: Microsatellites for maritime surveillance - an update on the Norwegian smallsat program. In: Proceedings of the 69thInternational Astronautical Congress (IAC), Bremen, Germany, 1-5 October 2018 (2018) (IAC-18.B4.4.2x45204)

5. Burzigotti, P., Ginesi, A., Colavolpe, G.: Advanced receiver design for satellite-based automatic identification system signal detection. Int. J. Satell. Commun. Network. 30, 52-63 (2012). https:// doi.org/10.1002/sat.1007

6. Hassani, A., Lazaro, F., Plass, S.: An Advanced AIS Receiver using a Priori Information. In: Proceedings of the OCEANS 2015 conference, Genova, Italy (2015).https://doi.org/10.1109/OCEAN S-Genova.2015.7271475

7. Clazzer, F., Lázaro, F., Plass, S.: Enchanced AIS receiver design for satellite reception. CEAS Space J 8, 257-268 (2016). https:// doi.org/10.1007/s12567-016-0122-8

8. Dahl, O. F. H.: Space-Based AIS receiver for maritime traffic monitoring using interference cancellation. M. S. thesis, 
Norwegian University of Science and Technology, Department of Electronics and Telecommunications (2006)

9. Helleren, Ø., Olsen, Ø., Berntsen, P. C., Strauch, K., Alagha, N.: Technology reference and proof-of-concept for a space-based Automatic Identification System for maritime security. In: Proceedings of the 4S Symposium, Rhodes, Greece (2008)

10. Picard, M., Oularbi, M.R., Flandin, G., Houcke, S.: An Adaptive Multi-User Multi-Antenna Receiver for Satellite-Based AIS Detection. In: Proceedings of the Advanced Satellite Multimedia Systems Conference (ASMS) and the 12thSignal Processing for Space Communications Workshop (SPSC), pp. 273-280 (2012). https://doi.org/10.1109/ASMS-SPSC.2012.6333088

11. Maggio, F., Rossi, T., Cianca, E., Ruggieri, M.: Digital beamforming techniques applied to satellite-based AIS receiver. IEEE Aerosp. Electron. Syst. Mag. 29(6), 4-12 (2014). https://doi. org/10.1109/MAES.2014.130168

12. Zhou, M., van der Veen, A.-J., van Leuken, R.: Multi-user LEOsatellite receiver for robust space detection of AIS messages. In: Proceedings of the 2012 IEEE Conference on Acoustics, Speech and Signal Processing (ICASSP), pp. 2529-2532 (2012). https:// doi.org/10.1109/ICASSP.2012.6288431

13. Carson-Jackson, J.: Satellite AIS - developing technology or existing capability? J. Navigation 65(2), 303-321 (2012). https://doi. org/10.1017/S037346331100066X

14. Posada, M., Greidanus, H., Alvarez, M., Vespe, M., Cokacar, T. and Falchetti, S.: Maritime awareness for Counter-Piracy in the Gulf of Aden. In: Proceedings of 2011 International Geoscience \& Remote Sensing Symposium, pp. 249-252 (2011). https://doi. org/10.1109/IGARSS.2011.6048939

15. Dembovskis, A.: Testbed for performance evaluation of SAT-AIS receivers. In: Proceedings of the Advanced Satellite Multimedia Systems Conference (ASMS) and the 12thSignal Processing for Space Communications Workshop (SPSC), pp. 253-257 (2012). https://doi.org/10.1109/ASMS-SPSC.2012.6333085

16. Nakamura, Y., Nishijo, K., Murakami, N., Kawashima, K., Horikawa, Y., Yamamoto, K., Ohtani, T., Takhashi, Y., Inoue, K.: Small Demonstration Satellite-4 (SDS-4): Development, Flight Results, and Lessons Learned in JAXA's Microsatellite Project. In: Proceedings of the AIAA/USU Conference on Small Satellites, the Year in Review, SSC13-X-1 (2013). https://digitalcommons. usu.edu/smallsat/2013/all2013/113/. Accessed 5 Apr 2019

17. International Telecommunication Union (ITU): Recommendation ITU-R M.1372-5-technical characteristics for an automatic identification system using time division multiple accessing the VHF maritime mobile frequency band (2014)

18. International Telecommunication Union (ITU): Recommendation ITU-R M.585-7-assignment and use of identities in the maritime mobile service (2015)

19. Eriksen, T., Høye, G., Narheim, B., Meland, B.J.: Maritime traffic monitoring using a space-based AIS receiver. Acta Astronaut. 58(10), 537-549 (2006). https://doi.org/10.1016/j.actaa stro.2005.12.016

20. Skauen, A.N., Olsen, Ø.: Signal environment mapping of the automatic identification system frequencies from space. Adv. Space Res. 57(3), 725-741 (2015). https://doi.org/10.1016/j. asr.2015.12.002
21. International Telecommunication Union (ITU): Recommendation ITU-R M.2169-improved satellite detection of AIS (2009)

22. European Space Agency (ESA): Atlantis leaves Columbus with a radio eye on Earth's sea traffic (2009). https://www.esa.int/Our_ Activities/Operations/i_Atlantis_i_leaves_Columbus_with_a_ radio_eye_on_Earth_s_sea_traffic. Accessed 22 Feb 2019

23. Olsen, Ø., Agersborg, J. A., Helleren, Ø., Skauen, A. N., Storesund, F. A. S.: AISSat-1-Happily working overtime. In: Proceedings of the 4S Symposium Port Petro, Majorca Island, Spain, May 26-30, 2014 (2014)

24. Bradbury, L. M., Diaconu, D., Laurin, S. M., Ma, C., Beattie, A., Spydevold, I., Haugli, H., Zee, R., Harr, J., Udnæs, F.: NorSat-2: Enabling Advanced Maritime Communications with VDES. In: Proceedings of the AIAA/USU Conference on Small Satellites, Next on the Pad 2, SSC17-XIII-08 (2017). https://digitalcom mons.usu.edu/smallsat/2017/all2017/167/. Accessed 5 Apr 2019

25. Pehradnia, P., Beattie, A., Kekez, D., Zee, R., Walter. B., Koller, S., Pfiffner, D., Finsterle, W.: NorSat-1: Enabling high performance and multipurpose microsatellite missions. In: Proceedings of the AIAA/USU Conference on Small Satellites, Year in review, SSC18-I-06 (2018). https://digitalcommons.usu.edu/small sat/2018/all2018/256/

26. European Space Agency (ESA): Novel SAT-AIS Receiver Phase $B 2 / C / D$ (2015). https://artes.esa.int/projects/novel-sat-ais-recei ver-phase-b2cd. Accessed 21 Mar 2019

27. Bråten, L. E., Eriksen, T., Skauen, A. N., Bjørnevik, A., Haugli, H. C., Løge, L.: On the VHF radio channel for the data exchange system via satellite (VDE-SAT): experimental results from the NorSat-2 satellite experiment. In: Proceedings of the $36^{\text {th }}$ International Communications Satellite Systems Conference (ICSSC), Niagara Falls, Canada, October 15-18 2018 (2018).

28. Skauen, A.N.: Quantifying the tracking capability of space-based AIS systems. Adv. Space Res. 57(2), 527-542 (2015). https://doi. org/10.1016/j.asr.2015.11.028

29. Li, S., Chen, L., Chen, X., Zhao, Y., Yang, L.: Statistical analysis of the detection probability of the TianTuo-3 space-based AIS. J. Navigation 71(2), 467-481 (2018). https://doi.org/10.1017/S0373 463317000649

30. Eriksen, T., Greidanus, H., Alvarez, M., Nappo, D., Gammieri, V.: Quality of AIS Services for Wide-Area Maritime Surveillance. In: Proceedings of MAST 2014 Conference, Istanbul, 21 st May 2014. Session: Surface, Maritime Security 3: Surveillance/ Domain Awareness (2014)

31. Høye, G.: Ship Detection Probability Analysis for a Possible Long-range AIS System. FFI/RAPPORT-2004/04383 (2004). https://www.ffi.no/no/Rapporter/04-04383.pdf. Accessed 5 Apr 2019

32. Olsen, Ø.: Global vessel traffic model. FFI/RAPPORT-2012/00048 (2012). https://www.ffi.no/no/Rapporter/12-00048.pdf. Accessed 5 Apr 2019

Publisher's Note Springer Nature remains neutral with regard to jurisdictional claims in published maps and institutional affiliations. 